\title{
Enhanced Visualization of Polysaccharides from Aqueous Suspensions
}

\author{
Charles-Philippe Lienemann, Denis Mavrocordatos, and Didier Perret* \\ Institut de Chimie Minérale et Analytique, Université de Lausanne, BCH, CH-1015 Lausanne, Switzerland
}

\begin{abstract}
Aqueous suspensions of polysaccharides such as those prepared for domestic and industrial applications or present in natural waters, although difficult to visualize by conventional transmission electron microscopy (TEM) because of their poor electron density, can be characterized at the ultrastructural level by using mild en bloc staining and contrast enhancement by energy-filtered TEM (EF-TEM). The advantages and drawbacks of the proposed method are discussed in relation to the different parameters controlling the quality of final images. It is shown, with synthetic polysaccharides, purified algal fibrils and lacustrine exocellular polymers as key examples, that optimizing specimen preparation and visualization parameters allows unbiased identification of organic substructures never revealed or strongly degraded by classical microscopic procedures.
\end{abstract}

Key words: fibrillar polysaccharides, contrast enhancement, energyfiltered transmission electron microscopy (EF-TEM), densitometry, stain, uranyl acetate.

Carbohydrates in nature show high diversity and complexity in their composition, structure and function $[1,2]$. They are produced as neutral or acidic compounds by all living species, from micro-organisms to higher animal cells, and it is not surprising to detect them in our environment (soils, waters), as a consequence of exocellular release (extracellular polysaccharides, EPS) or degradation process in the natural reservoirs [3].

Because of their thickening properties, native or derivatized polysaccharide biopolymers from botanical and microbial sources have found widespread

\footnotetext{
* To whom correspondence should be addressed
}

applications for industrial, domestic and environmental uses where rheology, gelling or pseudoplasticity is of concern $[4,5]$.

The behaviour of polysaccharides in synthetic formulations and natural suspensions will primarily depend on their macromolecular structure, varying from totally linear (cellulose) to strongly branched (spherocolloids like amylopectin or gum arabic) compounds; due to minimal steric hindrances, the former have a strong tendency to pack into water-insoluble thick fibres, while the latter are usually highly hydrated (hydrocolloids) and form gels which can contain up to 99.5\% water [6].

Polysaccharides have a high complexing capacity for di- and trivalent ions $[7,8]$. Furthermore, the hydroxyl groups of polysaccharides tend to interact with the hydrated surfaces of dispersed mineral particles (oxyhydroxides) and with hydrophilic organic material by hydrogen binding, and to form biofilms on submerged surfaces $[9,10]$. These properties are demonstrated in soils, where fungal polysaccharides act as binding/aggregating agents between clay particles and as water stabilizers [11-13]. Polysaccharides are used in water treatment plant for the removal of undesirable substances by cross-linking, micro-gel formation and flocculation. However, the role of EPS on the stability of suspended mineral particles and on the transport processes of micropollutants in freshwaters and marine environments still remains unclear, mainly because of a lack of information on the structure of polysaccharidic fibrils at the $\mu \mathrm{m}$ to $\mathrm{nm}$ scale.

In order to address this lack, the present study focuses on the energy-filtered transmission electron microscopic (EF-TEM) tools used for the identification and visualization of fibrillar polysaccharides (hydrocolloids) in synthetic or natural media, a domain 
scarcely studied before. Amongst others, we have used uranyl acetate stain under carefully selected conditions and optimized energy-filtered visualization of the resulting electron-contrasted entities. This provides valuable and reproducible information on the architecture of carbohydrate fibrils, even at the ultrastructural level, without introducing artefacts.

\section{Experimental}

\section{Polysaccharides}

Synthetic suspensions of polysaccharides were prepared with ultrapure ultrafiltered UV-irradiated water (Elgastat UHQ-II). Experiments were carried out under a laminar-flow hood in order to avoid contamination on TEM grids. Three different sources of polysaccharides were used as models: Commercial xanthan, purified algal fibrils and natural aquatic suspensions.

Xanthan (MW >2 $\times 10^{6}$ dalton; Flocon 4800 C, courtesy of Pfizer), which is widely used in oil recovery procedures, is a concentrated gel (ca. $90 \mathrm{~g} / 1$ ) produced by fermentation of Xanthomonas campestris. Dilute suspensions of this polysaccharide (ca. $0.2 \mathrm{mg} / 1$ ) were stirred for $24 \mathrm{~h}$ prior to use.

The second source is a purified algal suspension of Scenedesmus acutus (hereafter refered to as "isolated fibrils") at a mature stage of fibril production ([14], strain B4; gift from Prof. J. Kushner, University of Toronto). Polysaccharides were isolated by centrifugation of the crude suspension ( $3500 \mathrm{rpm}, \mathrm{RCF}=2500 \mathrm{~g}, 15 \mathrm{~min}$ ) in order to separate cells from free fibrils. The concentration of polyuronic acids [15] in the initial crude suspension was below $20 \mu \mathrm{mol} / \mathrm{ml}$, and analyses of the supernatant (free fibrils) yielded $2 \mathrm{mg} / 1$ of Total Organic Carbon (Shimadzu 5000; ca. $5 \mathrm{mg} / 1$ when converted into $\mathrm{C}_{6} \mathrm{H}_{12} \mathrm{O}_{6}$ ).

For several experiments, monodisperse haematite microparticles $\left(\alpha-\mathrm{Fe}_{2} \mathrm{O}_{3}, 70 \pm 5 \mathrm{~nm} ; 0.1 \mathrm{mg} / \mathrm{l}\right)$ were added as model mineral microparticles to xanthan or isolated fibrils $(0.2 \mathrm{mg} / \mathrm{l})$ in order to simulate mineral-organic networks observed in natural systems $[12,16,17]$.

Natural aquatic samples containing polysaccharides were collected in surface waters $(0-20 \mathrm{~m})$ of Lake Lugano (Ticino, Switzerland) during the 1994 summer season (higher concentrations of photosynthetic products). Continuous precentrifugations were directly processed on shore in order to eliminate particles with sizes larger than $1 \mu \mathrm{m}$ and densities higher than $1.2 \mathrm{~g} / \mathrm{cm}^{3}(11000 \mathrm{rpm}$, $\mathrm{RCF}=9500 \mathrm{~g}$ ).

\section{Preparation of TEM Specimens}

A $0.2 \mu \mathrm{m}$ filtered, $2 \%$ aqueous stock solution of uranyl acetate was used to stain the polysaccharides. Small aliquots of this solution were added directly to the suspensions of polysaccharides (en bloc staining [18]) prior to preparation of TEM specimens in order to obtain the desired stain concentrations.

Specimens were prepared on TEM grids ( $\mathrm{Cu} 200$ mesh; collodion covered, carbon coated) by a non-disturbing ultracentrifugation procedure described elsewhere [17]. Suspensions were directly ultracentrifuged above TEM grids in a swing-out rotor $(17000 \mathrm{rpm}$, $\mathrm{RCF}=50000 \mathrm{~g}, 15 \mathrm{~min}$ ). After removal of supernatant and recovery of grids, post-coating of specimens was achieved by adding $5 \mu \mathrm{l}$ of a dilute Nanoplast FB101 resin (hydrophilic embedding melamine resin [19]; resin: $\mathrm{H}_{2} \mathrm{O}=1: 10$ ) on grids deposited on the horizontal disk of a home-made microcentrifuge (10 s centrifugation at 7000 rpm [20]).
Analytical Electron Microscopy

Preliminary observations were done on a medium-resolution TEM (Zeiss EM10;80 keV). After selection of regions of interest, analytical electron microscopy of specimens was achieved with a high-resolution energy-filtered TEM (EF-TEM; Zeiss CEM902 equipped with a Castaing-Henry filter and a TV camera; $80 \mathrm{keV}$ ).

The EF-TEM discriminates elastically and inelastically forwardscattered electrons that have lost discrete amounts of energy $\left(\Delta \mathrm{E}_{10 \mathrm{~s} s}\right)$ during their interaction with the specimen; a series of characteristic $\Delta \mathrm{E}_{\text {loss }}$ edges arises for each element present in the analysed specimen [21-23]. In previous studies $[18,24]$ we have shown with aquatic suspensions (mineral particles and organic matter) that EF-TEM can be applied to $(i)$ elemental analysis (spectrum mode; electron energy loss spectrometry, EELS-TEM), (ii) elemental mapping (imaging mode; electron spectroscopic Imaging, ESI-TEM), and (iii) the visualization of organic matter (structure-sensitive mode; contrast tuning, CT-TEM). The last technique has been selected and optimized in this work to enhance contrasting of polysaccharide fibrils.

Densitometric measurements of TEM prints were carried out with Wincam, a software dedicated to analysis of thin layer chromatography plates, after digitizing of images recorded with a CCD camera (Cybertech CS-1).

\section{Results and Discussion}

Contrast enhancement of poorly electron-dense organic matter by conventional TEM can be achieved when heavy metal compounds are used as stains $[25,26]$. However, ultimate visualization of ultrastructures of fine polysaccharidic fibrils without producing artefact is usually difficult to achieve, mainly because of non-specific precipitation of stains when the aqueous matrix of the sample is complex [27]. On the other hand, it has been observed that contrast enhancement of EF-TEM is improved with stained specimens when visualized in CT-TEM mode below the carbon $K$ ionisation edge [23].

Figure 1 is a spectacular example of the increase in contrast which is obtained by CT-TEM under optimal conditions for a synthetic network of slightly stained polysaccharides. Below the carbon ionization edge, the increase in contrast is produced by the quantitative difference in number of electrons scattered from $(i)$ the supporting film and (ii) heavy metal enriched organic material (higher atomic mass) plus the supporting film.

The advantages of CT-TEM for this kind of specimen are clearly demonstrated; while ultrafine fibrils (see arrows) are hardly, if at all, visible in the conventional image, they are sharply revealed in the filtered image. It is however necessary to determine (i) the optimal energy $\Delta \mathrm{E}_{\text {loss }}$ where contrast enchancement of organic matter is obtained and (ii) the concentration of stain leading to unbiased fibril architecture. 

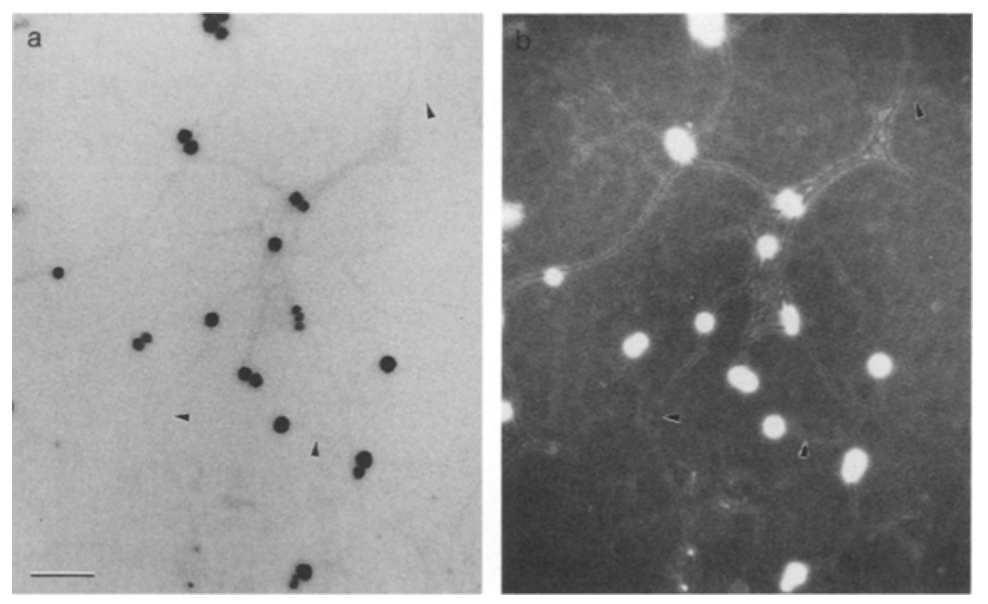

Fig. 1. Bright field TEM (a) and CT-TEM (b; acquired at $\Delta \mathrm{E}_{\text {loss }}=250 \mathrm{eV}$ ) of a synthetic mixture of xanthan polysaccharides $(0.2 \mathrm{mg} / \mathrm{l})$ and haematite microparticles $(0.1 \mathrm{mg} / \mathrm{l})$ prepared by ultracentrifugation of suspension with en bloc staining (uranyl acetate $0.1 \%$ ). Because of the procedure used in CT-TEM for the contrast enhancement of organic fibrils, microparticles appear as white blurred spots on the images. $\operatorname{Bar}=0.5 \mu \mathrm{m}$

CT-TEM images have been acquired between $\Delta \mathrm{E}_{\text {loss }}=0 \mathrm{eV}$ and $\Delta \mathrm{E}_{\text {loss }}=300 \mathrm{eV}$ with an energy resolution of $15 \mathrm{eV}$; this range includes the carbon $K$ ionization edge $(284 \mathrm{eV})$. Xanthan and isolated fibrils have been used for this optimization in order to determine the effect of the nature of species on the optimum $\Delta \mathrm{E}_{\text {loss }}$ value. A print was recorded at each $\Delta \mathrm{E}_{\text {loss }}$ and the differences in grey levels were measured densitometrically between the background surrounding selected fibrils and the fibrils themselves. The results of these measurements are presented in Fig. 2.

Small scale inhomogeneities in the background and in the fibrils explain the high variability within individual measurements (maximum 10\% for three selected segments along the fibrils). On the other hand, den-

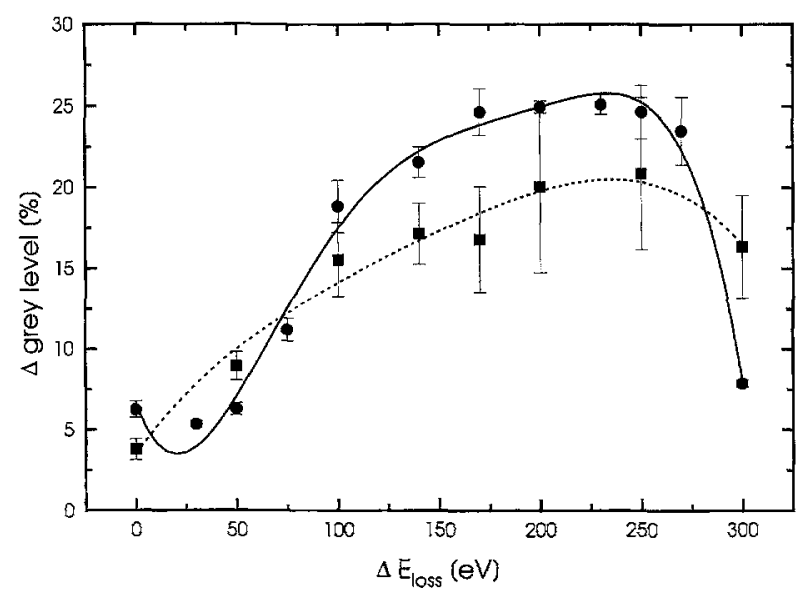

Fig. 2. Densitometric measurements of CT-TEM images of synthetic xanthan $(\bullet)$ and isolated fibrils $(\boldsymbol{C})$ recorded at different $\Delta \mathrm{E}_{\text {loss }}$ values. For each fibril, 3 measurements were achieved on different segments of the polysaccharide. Densitometric values are calculated as the difference in grey levels between the fibril segment and its surrounding. Error bars represent standard deviations sitometric measurements show a relatively large range of $\Delta \mathrm{E}_{\text {loss, }}$, between ca. $170 \mathrm{eV}$ and ca. $270 \mathrm{eV}$, where the contrast is higher. The optimal contrast is obtained between 230 and $250 \mathrm{eV}$ (around $250 \mathrm{eV}$ for isolated fibrils and around $230 \mathrm{eV}$ for xanthan).

These results indicate that the intimate composition of polysaccharides could slightly influence their contrast. The degree of interaction between stain and polysaccharides might also be responsible for the observed differences in contrast. These differences would be minimized with a lower energy resolution. Nonetheless, our results on individual fibrillar material are in agreement with observations on more classical tissue sections of biological samples $[28,29]$.

Figure 3 shows the drastic differences in contrast between the background (collodion and carbon film) and a network of slightly stained xanthan fibrils, as obtained by CT-TEM at different energies.

The thickness of material under the electron beam is usually an important factor for proper EF-TEM analysis [30]. We have, however, observed that removal of the collodion supporting film by solvent dissolution did not increase the quality of the final filtered image for stained and unstained specimens. This is explained by the negligible mass effect of the very thin (ca. 2-6 nm, measured according to [21]) and poorly electron-dense collodion film, as compared with the stain-loaded fibrils (ca. $5-10 \mathrm{~nm}$ ) deposited on the carbon film (ca. 6-10 nm).

The above results show that CT-TEM is a valuable and powerful method for the highly contrasted visualization of individual polysaccharide fibrils, but that observation of ultra-fine fibrils is not possible without the use of stains. Uranyl acetate is a non-specific marker usually employed to increase the contrast of 

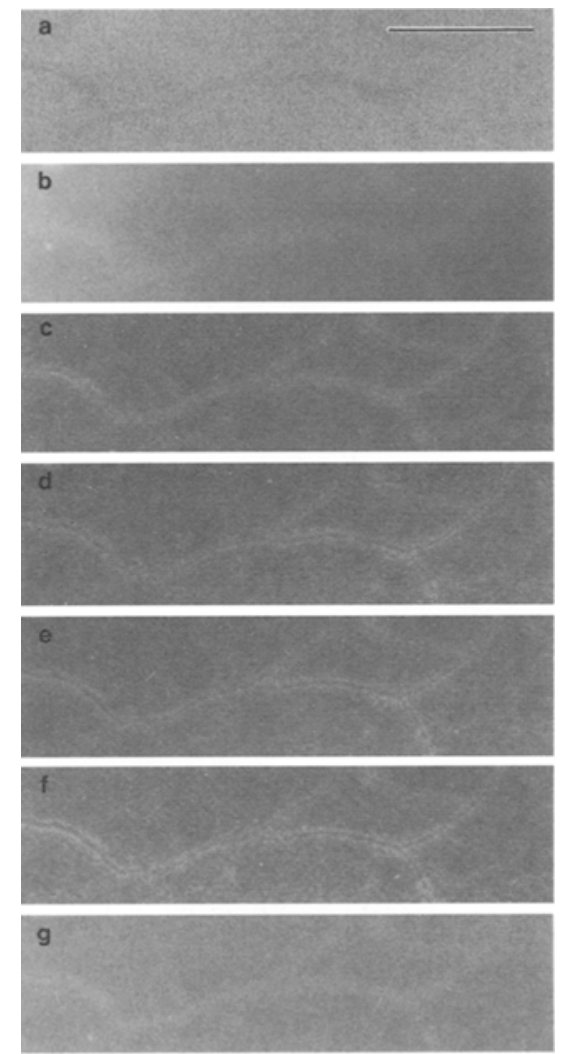

Fig. 3. Assembling of bright field TEM (a) and CT-TEM (b-g) of a network of xanthan (uranyl acetate $0.05 \%$ en bloc). b $50 \mathrm{eV}, \mathrm{c} 140$ $\mathrm{eV}, \mathbf{d} 200 \mathrm{eV}$, e $250 \mathrm{eV}$, f $270 \mathrm{eV}, \mathrm{g} 300 \mathrm{eV}$. Bar $=0.5 \mu \mathrm{m}$

TEM images for organic material in biological samples. The use of this stain at conventional concentrations $(1-2 \%)$ has, however, been shown to drastically hamper the detection of the ultrastructure of polysaccharides [27], because of the formation of grainy deposits along the fibrils, masking nodes and intersections within networks.

It is thus necessary to determine the optimum concentration of stain which fulfils the compromise between high CT-TEM contrast and low deleterious effect of stain. Concentrations between 0.5 and $0.01 \%$ were added to suspensions for en bloc staining just prior to ultracentrifugation above grids. Comparison of conventional TEM and CT-TEM results, illustrated in Fig. 4, shows that the compromise is optimal between 0.1 and $0.05 \%$ final stain concentration, where the ultrastructure of polysaccharides is maintained on CT-TEM images. At lower stain concentrations, fibrils are difficult to visualize by conventional TEM, while grainy deposits are observed at higher concentrations.
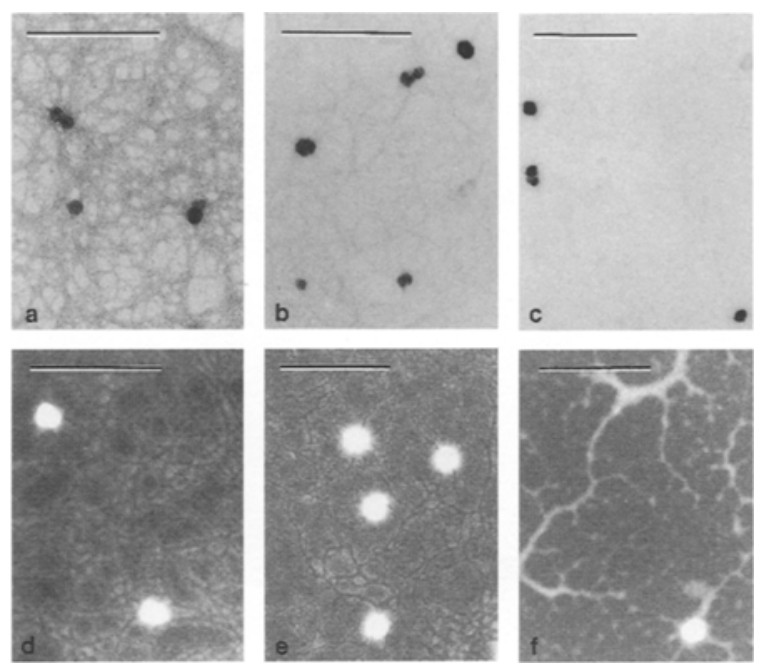

Fig. 4. Bright field TEM (a-c) and CT-TEM (d-f) images of xanthanhaematite specimens prepared by en bloc staining with uranyl acetate at $0.2 \%(\mathbf{a}), 0.1 \%(\mathbf{b}, \mathbf{d}), 0.05 \%(\mathbf{c}, \mathbf{e})$ and $0.01 \%(\mathbf{f}) . \mathrm{Bar}=0.5 \mu \mathrm{m}$

For stain concentrations below $0.01 \%$, only thick aggregates of fibrils are revealed (Fig. 4f).

When optimum staining conditions are chosen, polysaccharide fibrils are homogeneously and reproducibly contrasted. Figure 5 is a combination of optimal TEM (conventional image), CT-TEM (contrast enhancement of organic matter) and ESI-TEM (elemental mapping of uranium, recorded at the $O_{4,5}$ ionization edge of this element [31]). Uranyl stain, visualized as uranium on Fig. $5 \mathrm{c}$, is exclusively and smoothly distributed at the surface of fibrils, without non-specific deposits on the carbon film, thus validating the use of uranyl acetate stain at low concentration.

One might envisage using ESI-TEM images of the uranium stain instead of CT-TEM to record contrasted images of polysaccharide fibrils; however, such images result in a loss of information for ultra-fine substructures of fibrils and are more fastidious to acquire (risk of specimen drift during recording of background and edge images).

When studying fragile specimens, it is necessary to prevent them from being physically or chemically transformed prior to TEM investigation. For instance, natural suspensions sampled from anoxic water layers are highly redox sensitive; they are usually subject to fast air oxidation leading to precipitation of iron oxihydroxide particles from dissolved $\mathrm{Fe}^{2+}$, which causes factitious structures to appear. On the other hand, flexible polysaccharidic fibrils can shrink during air drying on specimen grids. In order to avoid biased 

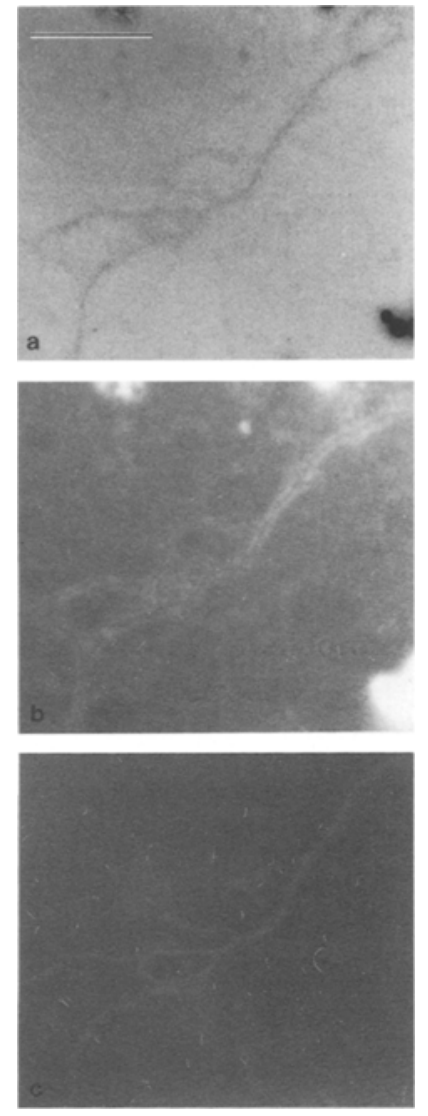

Fig. 5. Bright field TEM (a), CT-TEM (b; acquired at $\Delta \mathrm{E}_{\text {loss }}=250 \mathrm{eV}$ ) and ESI-TEM (c; acquired at uranium $O_{4,5}$ edge, $\otimes \Delta \mathrm{E}_{\text {loss }}=115 \mathrm{eV}$; subtracted background extrapolated from images acquired at $\Delta \mathrm{E}_{\text {loss }}=80 \mathrm{eV}$ and $90 \mathrm{eV}$ ) of a polysaccharide suspension with $0.1 \%$ uranyl acetate en bloc $\otimes$ staining. Bar $=0.5 \mu \mathrm{m}$

microscopic information, physico-chemical protection of specimen is achieved by post-coating of grids with hydrophilic Nanoplast resin. Figure 6 shows that
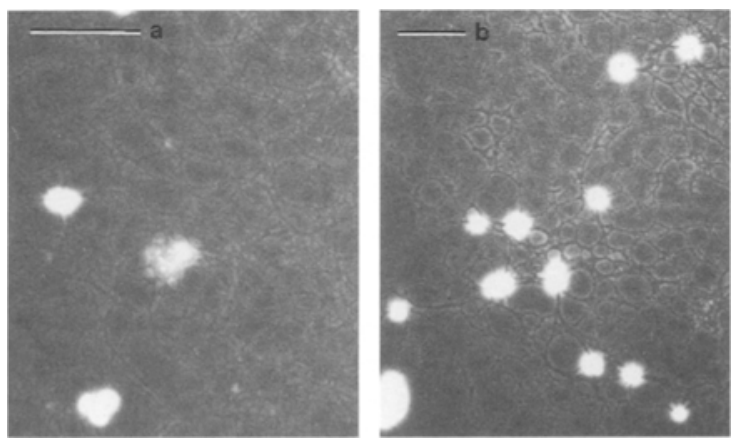

Fig. 6. Effect of hydrophilic Nanoplast embedding resin on the quality of CT-TEM images of xanthan $(0.2 \mathrm{mg} / \mathrm{l})$ and haematite $(0.1$ $\mathrm{mg} / \mathrm{l})$ mixtures $(0.1 \%$ uranyl acetate en bloc, staining). a Without and b with post-protective Nanoplast film. Bar $=0.5 \mu \mathrm{m}$
Nanoplast protective film does not lower the contrast of stained fibrils when they are visualized by CT-TEM.

The sum of the above results obtained on industrial xanthan fibrils demonstrate that mild staining procedures combined with the use of EF-TEM in contrast tuning mode lead to unbiased information on the architecture of this network-forming polysaccharide at the ultrastructural level. Our approach contributes and strengthens accurately documented studies on conformational and structural characterization of xanthan $[32,33]$. It is therefore also possible to demonstrate the applicaton of our proposed methods for the characterization and understanding of two other systems with different origins (purified algal suspensions; lacustrine samples).

In order to study the ability of freshwater algae to produce EPS and to understand the influence of trace metals on algal growth, fresh cultures of Scenedesmus acutus, were exposed to various concentrations of $\mathrm{Cu}$ and Ni (D. J. Kushner, personal communication). Polysaccharide fibrils produced by different strains of this alga were then isolated and studied by CT-TEM. The highiy contrasted images obained by CT-TEM highlight valuable features at the ultrastructural level (Fig. 7). For instance, small substructures are observed at high magnification along the fibrils with a periodicity of ca. $35 \mathrm{~nm}$. While the origin of these nanoexcrescences is currently unknown, their position along the chain could be the consequence of a ternary structure of individual sugar moieties. The systematic CT-TEM characterization of the morphology of algal fibrillar polysaccharides, currently under investigation, might
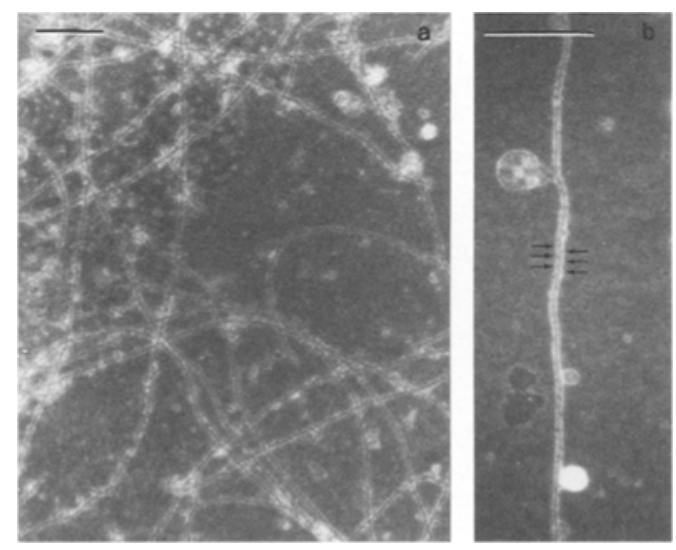

Fig. 7a, b. CT-TEM images of purified fibrils isolated from Scenedesmus acutus $(0.1 \%$ uranyl acetate en bloc staining; $\Delta \mathrm{E}_{\text {loss }}=250 \mathrm{eV}$ ). Arrows on $\mathbf{b}$ point to nanoexcrescences exhibiting a high spatial periodicity. $\mathrm{Bar}=0.5 \mu \mathrm{m}$ 
be useful to detect the effect of toxic metals on the behaviour of algae.

Natural aquatic samples have also been studied in order to validate our procedures. For this purpose, specimens from Lake Lugano were prepared for CTTEM visualization of native polysaccharides. Uranyl acetate was not added to aqueous suspensions, because organic matter of lacustrine or marine origin might already be naturally and slightly stained. This results from the complexation of dissolved cations (predominantly $\mathrm{Ca}^{2+}>\mathrm{Mg}^{2+}>\mathrm{Na}^{+}>\mathrm{K}^{+}$) by organic species [4].

When visualized by CT-TEM (Fig. 8), specimens exhibit highly contrasted networks of organic fibrillar material. Mainly because of technical limitations, these networks have rarely been observed or documented in natural waters, while CT-TEM allows an accurate description of their morphological properties at the ultrastructural level, even in the absence of stain. For instance, careful examination of Fig. 8 reveals that it is possible to distinguish between intimately connected (single arrows) and overlapping (double arrows) fibrils.

Combined with stereoscopic images (TEM equipped with a goniometer stage), CT-TEM should help in gaining a better insight into the complex 3-dimensional structure of organic networked macromolecules present in natural waters. Amongst others, one can clearly show by CT-TEM that mineral microparticles (clays, $\mathrm{SiO}_{2}, \mathrm{FeOOH}$ being the most relevant) strongly interact with EPS networks; evidence of these organo-

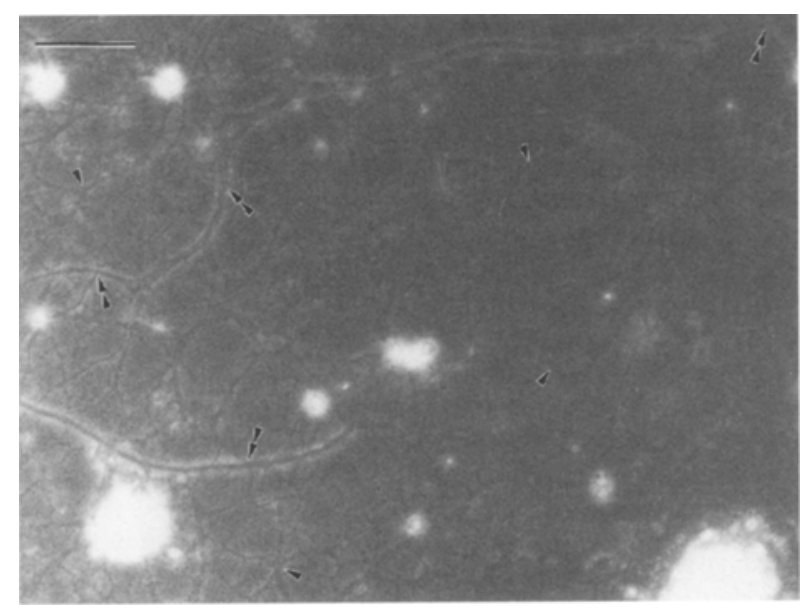

Fig. 8. CT-TEM image of Lake Lugano surface water polysaccharides in a heterogeneous mixture of mineral microparticles. Single arrows indicate naturally connected fibrils, while double arrows point to overlapping fibrils. $\mathrm{Bar}=0.5 \mu \mathrm{m}$ mineral entities in natural waters might explain inadequacies between field observations and theoretical predictions of (i) coagulation rate of microparticles (vs. stabilization by EPS) and (ii) adsorption of trace elements onto microparticles (vs. complexation by EPS moiety of organo-mineral entities).

\section{Conclusions}

EF-TEM visualization in contrast tuning mode allows identification of ultra-fine substructures of fibrillar polysaccharides in water suspensions, provided that these are labelled with a sufficient amount of marker. Uranyl acetate, a non-specific stain, can be used at low concentration to fulfil this requirement. When the ionic strength of the sample is low (i.e. synthetic suspensions) en bloc staining with uranyl acetate at ca. $0.05-0.1 \%$ prior to deposition of fibrils by ultracentrifugation on TEM grids has no deleterious effect on the morphology and thickness of polysaccharides while their network arrangements are retained. In the case of natural aquatic samples, visualization is even possible in the absence of stain. The highest contrast of fibrillar material is obtained at $\Delta \mathrm{E}_{\text {loss }}$ between 230 and $250 \mathrm{eV}$. Nanoplast post-protection of specimen can be effectively used in conjunction with CT-TEM imaging of polysaccharides without fading the contrast of micro-fibrils.

The proposed method can be usefully applied to natural and synthetic aqueous samples; it provides new opportunities for the physico-chemical characterization of fibrillar polysaccharides at the ultrastructural level, for a better understanding and modelling of their intimate architecture. The undisturbed characterization of polysaccharides in unstable and highly heterogeneous natural aquatic samples should contribute to studies on the importance of EPS in the control of the transport of mineral particulates and micropollutants, while simultaneously avoiding the strong structural modifications induced by physical or chemical bias in the usual procedures.

Acknowledgements. We gratefully acknowledge Prof. D. J. Kushner (University of Toronto, Canada) for providing fresh algal cultures, Dr. S. Fakan and his team (Center of Electron Microscopy, University of Lausanne) for access on EM facilities, and E. Leipzig-Pagani (Institute of Inorganic and Analytical Chemistry, University of Lausanne) for his help on densitometric measurements. This work was supported by grants from the Swiss National Science Foundation (project 21-36593.92) and the Swiss Prioritary Program Environment (Project Dytrac 50-39145.93) 


\section{References}

[1] A. Varki Glycobiology 1993, 3,97.

[2] G. G. Leppard, Sci. Tot. Environ. 1995, 165, 103.

[3] J. Buffle, Complexation Reactions in Aquatic Systems, an Analytical Approach, Ellis Horwood, Chichester, 1988.

[4] P. A. Sandford, J. Baird, in: The Polysaccharides, Vol. 2, (G. O. Aspinal, ed.), Academic press, New York, 1983, p.411.

[5] R. L. Whistler, J. N. BeMiller, Industrial Gums, 2nd Ed., Academic Press, New York, 1973.

[6] D. A. Rees, in: Advances in Carbohydrate Chemistry and Biochemistry, Vol. 24, (M. L. Wolfrom, R. S. Tipson, eds.), Academic press, New York, 1969, p.267.

[7] D. Cozzi, P. G. Desideri, L. Lepri, G. Ciantelli, J. Chromatogr. 1968, 35, 396.

[8] D. Cozzi, P. G. Desideri, L. Lepri, G. Ciantelli, J. Chromatogr. 1968, 35, 405 .

[9] D. G. Allison, Microbiol. Europe, 1993, Nov.-Dec., 16.

[10] H. C. Jones, I. L. Roth, W. M. Sanders, J. Bacteriol. 1969, 99, 316.

[11] C. Chenu, Soil Biol. Biochem. 1989, 21, 299.

[12] C. Chenu, Geoderma 1993, 56, 143.

[13] R. N. Yong, D. Mourato, Can. Geotech. J. 1990, 27, 774.

[14] T. Strycek, J. Acreman, A. Kerry, G. G. Leppard, M. V. Nermut, D. J. Kushner, Microb. Ecol. 1992, 23, 53.

[15] T. Bitter, H. M. Muir, Anal. Biochem. 1962, 4, 330.

[16] M. Fletcher, G. D. Floodgate, J. Gen. Microbiol. 1973, 74, 325.

[17] D. Perret, M. E. Newman, J.-C. Nègre, Y. Chen, J. Buffle, Wat. Res. 1994, 28, 91.

[18] D. Mavrocordatos, C.-P. Lienemann, D. Perret, Mikrochim. Acta 1994, 117, 39.

[19] D. Frösch, C. Westphal, Electron Microsc. Rev. 1989, 2, 231.
[20] D. Perret, G. G. Leppard, M. Müller, N. Belzile, R. DeVitre, J. Buffle, Wat. Res. 1991, 25, 1333.

[21] R. F. Egerton, Electron Energy-Loss Spectroscopy in the Electron Microscope, Plenum, New York, 1986.

[22] J. Fink, in: Advances in Electronics and Electron Physics, Vol. 75 (P. W. Hawkes, ed.), Academic Press, San Diego, 1989, p.121.

[23] L. Reimer, I. Fromm, P. Hirsch, U. Platte, R. Rennekamp, Ultramicroscopy, 1992, 46, 335.

[24] D. Perret, C.-P. Lienemann, D. Mavrocordatos, Microsc. Microanal. Microstruct. 1995, 6, 41.

[25] G. G. Leppard, in: Environmental Particles, Vol. 1. (J. Buffle, H. P. Van Leeuwen eds.), Lewis, Chelsea, 1992, p.231.

[26] A. W. Robards, A. J. Wilson, Procedures in Electron Microscopy, Wiley, Chichester, 1993.

[27] K. J. Wilkinson, S. Stoll, J. Buffle, Fresenius J. Anal. Chem. $1995,351,54$.

[28] C. W. J. Sorber, A. A. W. De Jong, N. J. Den Breejen, W. C. De Bruijn, Ultramicroscopy 1990, 32, 55.

[29] J. Colliex, C. Mory, A. L. Olins, D. E. Olins, M. Tence, $J$. Microsc. 1989, 153, 1.

[30] R. Door, K. D. Häberle, R. Martin, J. Microsc. 1994, 174, 183.

[31] L. Reimer, U. Zepke, J. Moesch, S. Schulze-Hillert, M. RossMessemer, W. Probst, E. Weimer, EEL Spectroscopy : a Reference Handbook of Standard Data for Identification and Interpretation of Electron Energy Loss Spectra and for Generation of Electron Spectroscopic Images, Carl Zeiss, Oberkochen, 1992.

[32] B. T. Stokke, A. Elgsaeter, O. Smidsrød, Int. J. Biol. Macromol. 1986, 8, 217.

[33] B. T. Stokke, A. Elgsaeter, D. A. Brant, S. Kitamura, Macromolecules 1991, 24, 6349.

Received September 23, 1995. Revision January 9, 1996. 